\title{
Vertebral fusion in two Early Jurassic sauropodomorph dinosaurs from the Lufeng Formation of Yunnan, China
}

Lida Xing, Bruce M. Rothschild, Hao Ran, Tetsuto Miyashita, W. Scott Persons IV, Toru Sekiya, Jianping Zhang, Tao Wang, and Zhiming Dong

Acta Palaeontologica Polonica 60 (3), 2015: 643-649 doi:http://dx.doi.org/10.4202/app.00001.2013

Here we describe two instances of pathological vertebral fusion in two genera of sauropodomorph dinosaurs from the Early Jurassic Lufeng Formation in Yunnan, China.

The first is a specimen, of Lufengosaurus huenei with two fused cervical vertebrae, and the other is a specimen of the Lufeng basal sauropod, with two fused caudal vertebrae. Both pathologies are consistent with spondyloarthropathy and represent the earliest known occurrence of that disease in dinosaurs. The two specimens affirm that early dinosaurs suffered from the same bone diseases as living vertebrates. Spondyloarthropathy in these dinosaurs may have been induced by long-term mechanical stress, such as weight bearing, and/or limited motion at the joint that would otherwise have inhibited such remodeling. In both cases, surface remodeling suggests that the animals survived well beyond the initiation of spondyloarthropathy.

Key words: Dinosauria, sauropodomorph, spondyloarthropathy, Jurassic, Lufeng Formation, China.

Lida Xing [xinglida@gmail.com] and Jianping Zhang [zhjping@cugb.edu.cn], School of the Earth Sciences and Resources, China University of Geosciences, Beijing 100083, China; Bruce M. Rothschild [bmr@ku.edu], Division of Vertebrate Paleontology, Natural History Museum and Biodiversity Research Center, University of

Kansas, 1345 Jayhawk Boulevard, Lawrence, KS 66045, USA; Hao Ran [ranhao.cn@ gmail.com ], Key Laboratory of Ecology of Rare and Endangered Species and Environmental Protection (Guangxi Normal University), Ministry of Education, Guilin 541004, China; Tetsuto Miyashita[tetsuto@ualberta.ca] and W.Scott Persons IV [persons@ualberta.ca ], Department of Biological Sciences, University of Alberta, 11455 Saskatchewan Drive, Edmonton, Alberta T6G 2E9, Canada; Sekiya Toru [t.sekiya.jlu@gmail.com ], Zigong Dinosaur Museum, Zigong 643013, Sichuan, China; Tao Wang [2625297725@qq.com ], Dinosaur Museum of World Dinosaur Valley Park, Lufeng, 651200, China; Zhiming Dong [1fdinodong@sina.com] Institute of Vertebrate Paleontology and Paleoanthropology, Chinese Academy of Sciences, Beijing 100044, China. 
This is an open-access article distributed under the terms of the Creative Commons Attribution License (for details please see creativecommons.org), which permits unrestricted use, distribution, and reproduction in any medium, provided the original author and source are credited.

Farif Full text $(560.3 \mathrm{kB})$ 